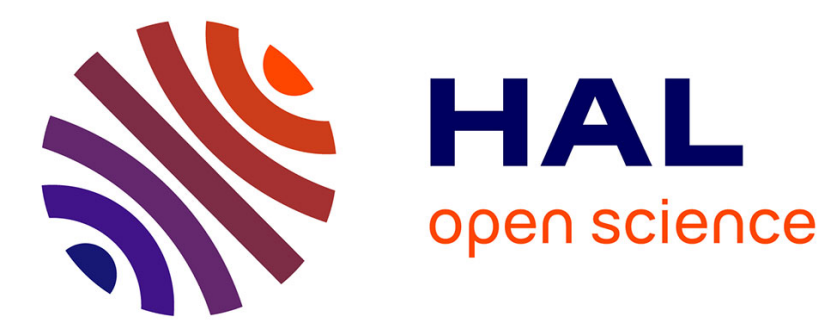

\title{
Spectral and Energy Efficient Fast-OFDM with Index Modulation for Optical Wireless Systems
}

\author{
Ali Waqar Azim, Marwa Chafii, Yannis Le Guennec, Laurent Ros
}

\section{To cite this version:}

Ali Waqar Azim, Marwa Chafii, Yannis Le Guennec, Laurent Ros. Spectral and Energy Efficient Fast-OFDM with Index Modulation for Optical Wireless Systems. IEEE Communications Letters, 2020, 24 (8), pp.1771 - 1774. 10.1109/LCOMM.2020.2989633 . hal-02869457

\section{HAL Id: hal-02869457 https://hal.science/hal-02869457}

Submitted on 16 Jun 2020

HAL is a multi-disciplinary open access archive for the deposit and dissemination of scientific research documents, whether they are published or not. The documents may come from teaching and research institutions in France or abroad, or from public or private research centers.
L'archive ouverte pluridisciplinaire HAL, est destinée au dépôt et à la diffusion de documents scientifiques de niveau recherche, publiés ou non, émanant des établissements d'enseignement et de recherche français ou étrangers, des laboratoires publics ou privés. 


\title{
Spectral and Energy Efficient Fast-OFDM with Index Modulation for Optical Wireless Systems
}

\author{
Ali Waqar Azim, Marwa Chafii, Yannis Le Guennec, and Laurent Ros
}

\begin{abstract}
This letter introduces fast-orthogonal frequencydivision multiplexing (FOFDM) with index modulation (IM) based on discrete cosine transform (DCT) for optical wireless systems. For intensity-modulation and direct detection, the proposed approach realizes a real-valued intensity waveform employing real-valued one-dimensional modulation formats and DCT; and brings about non-negativity via direct current bias. We shall establish that it attains up to $64 \%$ higher spectral efficiency (SE) with low order alphabets than conventional IM based on discrete Fourier transform and provides better flexibility to address the trade-off between $\mathrm{SE}$ and energy efficiency.
\end{abstract}

Index Terms-Discrete cosine transform, index modulation, intensity modulation-direct detection, optical wireless systems.

\section{INTRODUCTION}

$\mathbf{I}$ NTENSITY modulation and direct detection compatible optical-orthogonal frequency-division multiplexing (OOFDM) schemes for optical wireless systems (OWS), using complex exponentials as the orthogonal basis, have been scrupulously examined; with the forthright scheme being direct current (DC) O-OFDM (DCO-OFDM). An approach with an identical precept to attain a non-negative signal, but exercising (co)sinusoidal functions as orthogonal basis is DCO-Fast-OFDM (DCO-FOFDM) [1]. DCO-FOFDM enjoys some advantages over DCO-OFDM. First, sacrificing complex modulation formats $(\tilde{M}$-ary quadrature-amplitude modulation (QAM)) in favour of real ones ( $M$-ary pulse-amplitude modulation (PAM)) permits DCO-FOFDM exhibits natural Hermitian symmetry (HS) instead of imposing it by incorporating superfluous information on half of the subcarriers like in DCOOFDM. Second, in DCO-FOFDM, the subcarrier spacing reduces to half in comparison to DCO-OFDM, whilst preserving orthogonality [1]. This narrower inter-carrier spacing is beneficial in frequency selective environments as it leads to a flatter response per subcarrier [2].

It has been established in [3], [4] that coupling OFDM with index modulation (IM) adds flexibility in terms of spectral efficiency (SE) and energy efficiency (EE), may lessen peakto-average power ratio (PAPR), and impart robustness to inter-carrier interference. For OWS, a combination of IM with DCO-OFDM is investigated in [5], proceeding in DCOOFDM-IM. DCO-OFDM-IM have some shortcomings: (i) when $\tilde{M}=M^{2}$, it yields a minimal increase in SE over DCOOFDM/DCO-FOFDM, consequently, it may require higher order alphabets which degrade the EE; and (ii) it provides a limited EE/SE trade-off without improving EE. To circumvent these limitations, hereby, for the first time, we propose DCOFOFDM-IM by amalgamating IM with DCO-FOFDM. We

Ali Waqar Azim,Yannis Le Guennec and Laurent Ros are with Université Grenoble Alpes, CNRS, Institute of Engineering, Grenoble INP, GIPSALAB, 38000 Grenoble, France (email: aliwaqarazim@gmail.com, \{yannis.leguennec,laurent.ros\} @ grenoble-inp.fr).

Marwa Chafii is with ETIS, UMR 8051, Université Paris-Seine, Université Cergy-Pontoise, ENSEA, CNRS, 95000 Cergy, France (marwa.chafii@ensea.fr). shall demonstrate that DCO-FOFDM-IM has many concrete advantages over DCO-OFDM-IM and DCO-FOFDM: (i) substantially higher achievable SE and improved EE for low electrical signal-to-noise ratio (SNR) per bit; (ii) extended granularity to attain EE/SE trade-off; (iii) enhanced performance in time dispersive channels; and (iv) relaxed constraints on system hardware. We organize the rest of the article as follows. Section II analyzes the system model, presents some insights into the classical benchmarks and elucidates the transceiver design for DCO-FOFDM-IM. Section III assesses the system performance in terms of SE, EE/SE trade-off and the bit-error rate (BER) performance. Section IV renders the conclusions.

\section{A. Preliminaries}

\section{SYSTEM DESCRIPTION}

Consider DCO-OFDM-IM with $N$ subcarriers using complex exponentials, $\phi_{\tilde{n}}(t)=(1 / \sqrt{\tilde{T}}) e^{j 2 \pi \tilde{n} \Delta \tilde{f} t}, 0 \leq t<\tilde{T}, \tilde{n} \in$ $\llbracket-\tilde{N} / 2, \tilde{N} / 2-1 \rrbracket$ as orthogonal basis, where $\tilde{T}$ is the symbol period, and $\Delta \tilde{f}=1 / \tilde{T}$ is the inter-carrier spacing to satisfy orthogonality among the complex subcarriers, i.e., $\int_{0}^{\tilde{T}} \phi_{\tilde{n}}(t) \phi_{\tilde{n}^{\prime}}(t) \mathrm{d} t=0$ for $\tilde{n} \neq \tilde{n}^{\prime}$, whilst the integral evaluates to 1 if $\tilde{n}=\tilde{n}^{\prime}$. The single-sided base-band bandwidth is $\tilde{B}=(\tilde{N} / 2) \Delta \tilde{f}=\tilde{N} / 2 \tilde{T}[6]$. For implementation via discrete Fourier transform (DFT) and its inverse, the null and $-\tilde{N} / 2$ th frequencies are not modulated, the positive frequencies, i.e., $\tilde{n} \in \llbracket 1, \tilde{N} / 2-1 \rrbracket$ are modulated by $\tilde{M}$-ary QAM alphabets, while the negative frequencies, i.e., $\tilde{n} \in \llbracket-\tilde{N} / 2+1,-1 \rrbracket$ enforce HS. Thus, the subcarrier set for IM is $\tilde{\boldsymbol{\Theta}}=\llbracket 1, \tilde{N} / 2-$ $1 \rrbracket$ with cardinality $|\tilde{\boldsymbol{\Theta}}|=\tilde{N} / 2-1$. On the other hand, DCO-FOFDM-IM employs (co)sinusoidal functions, $\varphi_{n}(t)=$ $\sqrt{2 / T} \cos (2 \pi \Delta f n t), 0 \leq t<T, n \in \llbracket 0, N-1 \rrbracket$ as orthogonal basis, where $T$ is the symbol period, and $N$ is the number of subcarriers. An inter-carrier spacing of $\Delta f=1 / 2 T$ is needed to ensure orthogonality among the real-valued subcarriers, i.e., $\int_{0}^{T} \varphi_{n}(t) \varphi_{n^{\prime}}(t) \mathrm{d} t=0$ when $n \neq n^{\prime}$, with the integral equal to unity when $n=n^{\prime} \neq 0$. The single-sided bandwidth of DCOFOFDM-IM is $B=N \Delta f=N / 2 T$, which implies that the available set of subcarriers is $\Theta=\llbracket 0, N-1 \rrbracket$ with cardinality $|\Theta|=N$. DCO-FOFDM-IM is implemented using discrete cosine transform (DCT) and $M$-ary PAM. It may be noticed that $N / B T=\tilde{N} / \tilde{B} \tilde{T}=2$; and if we assume $\tilde{T}=T$, then $\Delta \tilde{f}=2 \Delta f$.

\section{B. Transceiver Design}

The total number of bits transmitted in a DCO-FOFDM-IM symbol of duration $T$ is

$$
\lambda=\underbrace{\left\lfloor\log _{2}\left(\begin{array}{l}
N \\
K
\end{array}\right)\right\rfloor}_{:=\lambda_{1}}+\underbrace{K \log _{2}(M)}_{:=\lambda_{2}},
$$

where $\lfloor\cdot\rfloor$ is the integer floor operator, $K$ is the number of active subcarriers per symbol, $\lambda_{1}$ and $\lambda_{2}$ are the index bits 


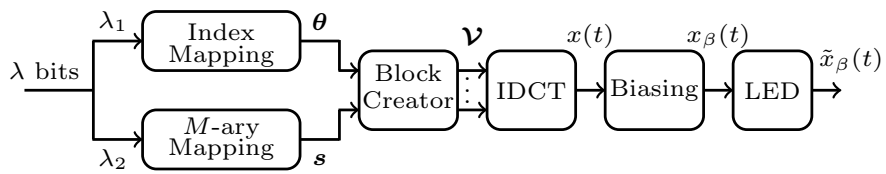

Fig. 1: Transmitter architecture for DCO-FOFDM-IM.

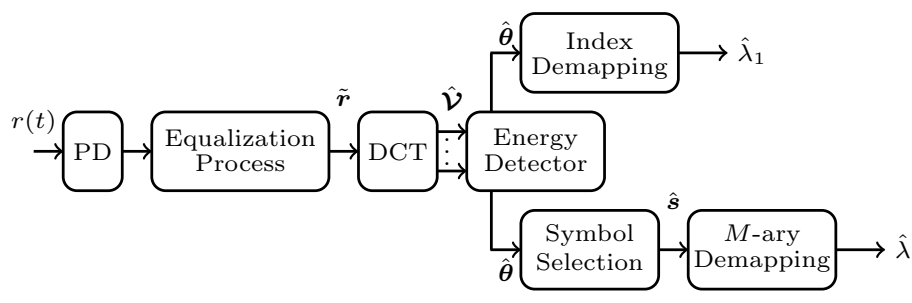

Fig. 2: Receiver configuration of DCO-FOFDM-IM.

mapped on to the index set of the activated frequency tones, and the modulation bits, respectively. Fig. 1 shows the transmitter structure of DCO-FOFDM-IM. The equiprobable bit sequence is split into $\lambda_{1}$ and $\lambda_{2}$. The index bits, $\lambda_{1}$ determine the subcarrier activation pattern (SAP), $\boldsymbol{\theta}=\left\{\theta_{0} \cdots \theta_{K-1}\right\} \in$ $\Theta$; which identifies $K$ out of $N$ subcarriers to be activated using combinatorial mapping [5]. The modulation bits, $\lambda_{2}$ are used to generate $K$ symbols, $\boldsymbol{s}=\left[s_{0} \cdots s_{K-1}\right]^{\mathrm{T}} \in \mathcal{S}_{M}$ to modulate on to the chosen SAP, $\boldsymbol{\theta}$. Here, $\mathcal{S}_{M}$ is the $M$-ary PAM constellation set, and $[\cdot]^{\mathrm{T}}$ denotes transposition, Using $\boldsymbol{\theta}$ and $s$, DCO-FOFDM-IM vector, $\mathcal{V}=\left[\mathcal{V}_{0} \cdots \mathcal{V}_{N-1}\right]^{\mathrm{T}}$ of length $N$ is created as

$$
\mathcal{V}_{n}=\left\{\begin{array}{ll}
s_{n}, & n \in \boldsymbol{\theta} \\
0, & \text { otherwise }
\end{array}, n \in \llbracket 0, N-1 \rrbracket .\right.
$$

Applying $N$-order IDCT, the multiplexed discrete signal, $x_{k}$ is obtained as

$$
x_{k}=\sqrt{\frac{2}{N}} \sum_{n=0}^{N-1} \mathcal{V}_{n} \alpha_{n} \cos \left[\frac{\pi n(2 k+1)}{2 N}\right], k \in \llbracket 0, N_{\mathrm{c}}-1 \rrbracket,
$$

where $\alpha_{n}=1 / \sqrt{2}$ for $n=0$ and $\alpha_{n}=1$ otherwise, $N_{\mathrm{c}}$ is the number of chips; which are equal to $N$. The electrical symbol energy and symbol power of $x_{k}$ is $\left\|x_{k}\right\|^{2}=$ $\sum_{k=0}^{N_{\mathrm{c}}-1} x_{k}^{2}=K E_{\text {avg }}$ and $\sigma^{2}=\left\|x_{k}\right\|^{2} / N$, respectively, where $\|\cdot\|^{2}$ evaluates Euclidean norm, $E_{\text {avg }}$ is the average $M$ ary PAM symbol energy; which is equal to $\left(M^{2}-1\right) / 3$. The discrete time series representation (3) is obtained from the analog counterpart $x(t)$ using $t=(2 k+1) T / 2 N$. A non-negative signal $x_{\beta}(t)$ is obtained after adding DC-bias equal to $\beta=\mu \sigma$, and clipping of residual negative amplitude excursions. Here, $\mu>0$ is a constant. On a decibel (dB) scale, the DCbias is $10 \log _{10}\left(\mu^{2}+1\right) \mathrm{dB}$. After electrical-to-optical (E/O) conversion (considering unity $\mathrm{E} / \mathrm{O}$ conversion factor), the optical intensity signal $\tilde{x}_{\beta}(t)$ is transmitted through an optical wireless channel with CIR $h(t)$.

The receiver architecture of the proposed approach is provided in Fig. 2. Considering perfect synchronization at the receiver, and ideal responsitivity of the photo-diode (PD) for optical-to-electrical conversion as conventionally done in the literature, the photo-detected electrical signal is contaminated by the ambient noise modeled as additive white Gaussian noise (AWGN) with single-sided power spectral density of $N_{0}$ resulting in received signal, $r(t)$. After discretization of $r(t)$, equalization process is performed in FD. DCO-FOFDM-IM requires additional DFT and inverse DFT (IDFT) to implement the equalization process [7]. After equalization, a discrete symbol vector $\hat{\boldsymbol{r}}=\left[\hat{r}_{0} \cdots \hat{r}_{N_{\mathrm{c}}-1}\right]^{\mathrm{T}}$ is obtained. After $N_{\mathrm{c}}$-order DCT, an estimated FOFDM-IM vector, $\hat{\mathcal{V}}=\left[\hat{\mathcal{V}}_{0} \cdots \hat{\mathcal{V}}_{N_{\mathrm{c}}-1}\right]^{\mathrm{T}}$ is obtained as

$$
\hat{\mathcal{V}}_{n}=\sqrt{\frac{2}{N}} \alpha_{n} \sum_{k=0}^{N_{\mathrm{c}}-1} \hat{r}_{k} \cos \left[\frac{\pi n(2 k+1)}{2 N}\right] .
$$

Subsequently, using $\hat{\mathcal{V}}$, the estimated SAP, $\hat{\boldsymbol{\theta}}$ is suboptimally identified by determining the energies of $N$ subcarriers, and then selecting the $K$ subcarriers with highest energies, similar to approach in [8]. Once $\hat{\boldsymbol{\theta}}$ is determined, the estimated index bits, $\hat{\lambda}_{1}$ are obtained. Symbol selector extracts the symbols on $\hat{\boldsymbol{\theta}}$, which are further processed to determine the modulation bits, $\hat{\lambda}_{2}$.

\section{Performance Analysis}

A. Spectral Efficiency Analysis

The foremost advantage of DCO-FOFDM-IM relative to DCO-OFDM-IM and DCO-FOFDM is its potential to achieve higher SE. For DCO-OFDM-IM and DCO-FOFDM, the number of bits transmitted per symbol is $\tilde{\lambda}=\left\lfloor\log _{2}\left(\begin{array}{c}\tilde{N} / 2-1 \\ \tilde{K}\end{array}\right)\right\rfloor+$ $\tilde{K} \log _{2}(\tilde{M})$, and $\bar{\lambda}=N \log _{2}(M)$, respectively. Moreover, the symbol duration and bandwidth for DCO-FOFDM is $\bar{T}$ and $\bar{B}=N / 2 \bar{T}$, respectively. The data rates in bits/s of DCO-FOFDM-IM, DCO-OFDM-IM and DCO-FOFDM are $R=\lambda / T, \tilde{R}=\tilde{\lambda} / \tilde{T}$ and $\bar{R}=\bar{\lambda} / \bar{T}$, respectively. Thus, the spectral efficiencies of the corresponding approaches in bits/s/Hz are appraised to be

$$
\begin{gathered}
\eta=\frac{R}{B}=\frac{2}{N}\left[\left\lfloor\log _{2}\left(\begin{array}{l}
N \\
K
\end{array}\right)\right\rfloor+K \log _{2}(M)\right], \\
\tilde{\eta}=\frac{\tilde{R}}{\tilde{B}}=\frac{2}{\tilde{N}}\left[\left\lfloor\log _{2}\left(\begin{array}{c}
\tilde{N} / 2-1 \\
\tilde{K}
\end{array}\right)\right\rfloor+\tilde{K} \log _{2}(\tilde{M})\right],
\end{gathered}
$$

and

$$
\bar{\eta}=\frac{\bar{R}}{\bar{B}}=2 \log _{2}(M) .
$$

For $\tilde{N}=N$, the binomial coefficient of DCO-FOFDM-IM is considerably larger than that of DCO-OFDM-IM because $|\Theta|>|\tilde{\Theta}|$, which results in higher SE.

\section{B. Maximization of Spectral Efficiency}

From (5) and (6) we know that $\eta$ (resp. $\tilde{\eta}$ ) depends on the number of active subcarriers, $K$ (resp. $\tilde{K}$ ). Thus, incurring to the fact that binomial coefficient is log-concave, it can have unique maximum relative to $K$ (or $\tilde{K}$ ) making it analytically conceivable to ascertain the approximate value of $K_{\text {approx }}$ (or $\tilde{K}_{\text {approx }}$ ) which maximizes $\eta$ (or $\tilde{\eta}$ ). The maximization of $\mathrm{SE}$ is equivalent to maximization of the number of bits per symbol. For brevity, we only consider DCO-FOFDM-IM for evaluation of $K_{\text {approx }}$. By dropping the floor function in (1) and taking its derivative with respect to $K$ gives

$$
\frac{\mathrm{d} \lambda}{\mathrm{d} K} \leq \frac{H_{N-K}-H_{K}+\log (M)}{\log (2)},
$$




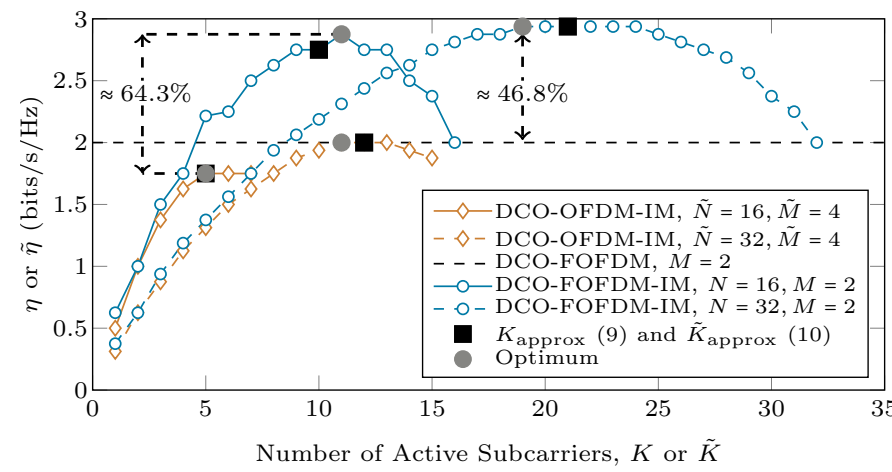

Fig. 3: Evolution of $\mathrm{SE}$ as a function of number of active subcarriers for DCO-FOFDM-IM and DCO-OFDM-IM.

where $H_{m}$ is the $m$ th harmonic number which is roughly equal to $H_{m} \approx \log (m)+\gamma$, where $\gamma$ is the Euler-Mascheroni constant. Setting (8) to zero and reintroducing the floor function leads to the following approximations of $K_{\text {approx }}$ [7]

$$
K_{\text {approx }} \approx\left\lfloor\frac{N M}{M+1}\right\rfloor .
$$

Similarly, for DCO-OFDM-IM, the approximation of $\tilde{K}_{\text {approx }}$ to attain maximum $\tilde{\eta}$ leads to

$$
\tilde{K}_{\text {approx }} \approx\left\lfloor\frac{(\tilde{N}-2) \tilde{M}}{2(\tilde{M}+1)}\right\rfloor .
$$

Fig. 3 illustrates the evolution of SE of DCO-FOFDM-IM (with $M=2$ ) and DCO-OFDM-IM (with $\tilde{M}=4$ ) by varying $K, \tilde{K}$ and using $N=\tilde{N} \in\{16,32\} . \eta$ for alphabet cardinality of $M$ is significantly higher than $\tilde{\eta}$ when $\tilde{M}=M^{2}$, e.g., considering $\{M, \tilde{M}, N, \tilde{N}\}=\{2,4,16,16\}, \eta$ is almost $64.3 \%$ higher than $\tilde{\eta}$. $\bar{\eta}$ for $M=2$ is 2 bits $/ \mathrm{s} / \mathrm{Hz}$, which is nearly $43.75 \%$ lesser than $\eta$. It is interesting to note that even if DCO-OFDM-IM uses twice the number of subarriers, i.e., $\tilde{N}=32$ versus $N=16, \eta$ remains higher than $\tilde{\eta}$. The term 'optimum' in the legend refers to the minimum value (of $K$ or $\tilde{K})$ at which the maximum SE of the respective approach can be attained. Note that $K_{\text {approx }}$ and $\tilde{K}_{\text {approx }}$ may not always be the optimal value of $K$ or $\tilde{K}$. The marginal discrepancy between approximate and optimal values is because the floor function is ignored to appraise the derivative of $\lambda$ or $\tilde{\lambda}$.

\section{Optimal Choice of Subcarriers/Active Subcarriers}

A certain value of $\eta$, depending on number of available subcarriers, is attainable with different combinations of $\{M, N, K\}$, e.g., $\eta$ of $1.75 \mathrm{bits} / \mathrm{s} / \mathrm{Hz}$ can be achieved by either $\{2,16,4\}$ or $\{2,32,7\}$. Ideally, $M$ should be kept as small as possible because the EE of DCO-FOFDM-IM degrades with high modulation order alphabets. To ascertain the best possible set $\{N, K\}$, we introduce a criterion called sparsity factor, $\psi$; which is the ratio of the active subcarrier to the total number of available subcarriers, i.e., $\psi=K / N$. The combination with the lowest sparsity factor should be the best choice. In the above case, $\psi=0.25$ when $\{2,16,4\}$ and $\psi=0.2188$ for $\{2,32,7\}$. Thus, the optimal pair $\{N, K\}$ is $\{32,7\}$. The optimal choice of $\{N, K\}$ impacts (i) the EE; and (ii) robustness against multipath time dispersive channel.
EE depends on the number of activated tones; as $\mathcal{V}$ is more sparse in case of optimal $\{N, K\}$, EE can be enhanced. Higher sparsity of $\mathcal{V}$ also leads to a diminished filtering impact of the channel on certain frequencies with a higher probability relative to DCO-OFDM/DCO-FOFDM. It is highlighted that to attain a target SE, the signal structure of DCO-FOFDMIM is more sparse in FD than DCO-OFDM-IM, e.g., to attain $1.75 \mathrm{bits} / \mathrm{s} / \mathrm{Hz}, \psi=0.2188$ for DCO-FOFDM-IM, whereas, for DCO-OFDM-IM, $\tilde{\psi}=2 \tilde{K} / \tilde{N}=0.5000$, when $\{\tilde{N}, \tilde{K}\}=\{32,8\}$. Consequently, DCO-FOFDM-IM is more energy efficient and is expected to be more robust against time dispersive propagation relative to DCO-OFDM-IM.

\section{Energy Efficiency and Bit-Error Performance}

In this subsection, we provide an overview of the performances of studied approaches by analyzing: (i) the evolution of $\mathrm{EE}$ with respect to $\mathrm{SE}$ in flat frequency channel; and (ii) BER performance as a function of electrical SNR per bit, $E_{\mathrm{b}_{\text {(elec) }}} / N_{0}$ in both flat frequency channel and a time dispersive channel (obtained via ceiling bounce model [9]). For impartial comparison, we consider the same detection method [8] for IM schemes. The clipping distortion should be minimal as it impacts SAP identification. Bias values are adjusted for each approach to attain a target BER with minimum required $E_{\mathrm{b}_{(\text {elec }}} / N_{0}$. At this stage, the analytical appraisal of optimal bias is beyond the purview of this article.

Fig. 4 illustrates the evolution of EE (required $E_{\mathrm{b}_{(\text {elec }}} / N_{0}$ for BER of $10^{-3}$ ) for the studied approaches with respect to SE. We consider $N=\tilde{N}=16, M \in\{2,4\}$ and $\tilde{M} \in\{4,16\}$. For DCO-FOFDM-IM, when $M=2, K=\llbracket 1,11 \rrbracket$ which varies $\eta=\{0.625 \rightarrow 2.875\}$ bits $/ \mathrm{s} / \mathrm{Hz}$ ( $K=10$ is skipped because it gives the same $\eta$ as for $K=9$ ). On the other hand, for $M=4, \eta>2.875 \mathrm{bits} / \mathrm{s} / \mathrm{Hz}$ is analyzed by considering $K=\llbracket 6,13 \rrbracket$, for which, $\eta=\{3 \rightarrow 4.375\}$ bits $/ \mathrm{s} / \mathrm{Hz}$. Both $K=11$ and $K=12$ results in $\eta=4.25 \mathrm{bits} / \mathrm{s} / \mathrm{Hz}$, therefore, $K=12$ is not used. In case of DCO-OFDM-IM, $\tilde{K}=\llbracket 1,5 \rrbracket$ and $\tilde{K}=\llbracket 3,7 \rrbracket$ are used for $\tilde{M}=4$ and $\tilde{M}=16$, respectively; which correspondingly changes $\tilde{\eta}=\{0.5 \rightarrow 1.75\}$ bits $/ \mathrm{s} / \mathrm{Hz}$ and $\tilde{\eta}=\{2.125 \rightarrow 3.5\}$ bits/s/Hz. Lastly, for DCO-FOFDM, the spectral efficiencies for $M=\{2,4\}$ are $\bar{\eta}=\{2,4\}$ bits/s/Hz. The values of bias used are indicated. From Fig. 4, we observe that (i) for $M=2$, DCO-FOFDM-IM is energy efficient compared to alternatives and provides higher number of operating points allowing more granularity when tradingoff EE and SE; (ii) DCO-OFDM-IM also provides limited $\mathrm{EE} / \mathrm{SE}$ trade-off, but, it is the least energy efficient option for spectral efficiences of $<3 \mathrm{bits} / \mathrm{s} / \mathrm{Hz}$; (iii) DCO-FOFDM is an appealing option for targeting higher spectral efficiencies, however, EE/SE trade-off is impossible; and (iv) the proposed approach has the potential to attain higher SE than others. We show that for target BER of $10^{-3}, E_{\mathrm{b}_{\text {(elec) }}} / N_{0}$ of $\approx 14$ $\mathrm{dB}$ permits $\mathrm{SE}$ of $2.5 \mathrm{bits} / \mathrm{s} / \mathrm{Hz}$ for the proposed technique versus $2 \mathrm{bits} / \mathrm{s} / \mathrm{Hz}$ and $1.875 \mathrm{bits} / \mathrm{s} / \mathrm{Hz}$, respectively, for DCOFOFDM and DCO-OFDM-IM. Additionally, a SE of $\approx 1.875$ bits $/ \mathrm{s} / \mathrm{Hz}$ is attained with an economy of almost $2 \mathrm{~dB}$ in $E_{\mathrm{b}_{\text {(elec) }}} / N_{0}$ compared to DCO-OFDM-IM. For $M=4$, though DCO-FOFDM-IM achieves higher SE; and has more operating points (for SE), it becomes less energy efficient making it only 


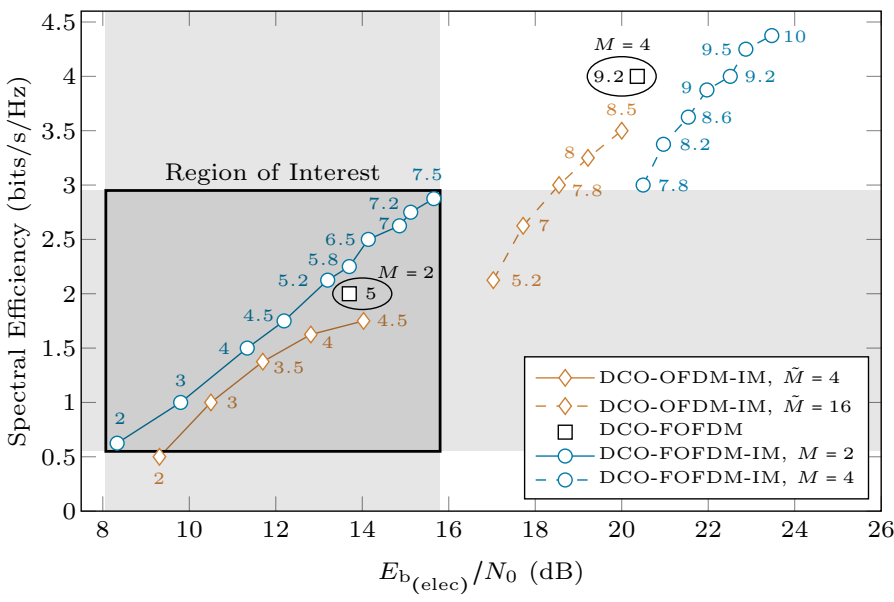

Fig. 4: The evolution of required $E_{\mathrm{b}_{(\text {elec }}} / N_{0}$ with respect to $\eta, \tilde{\eta}$ and $\bar{\eta}$ to achieve a BER of $10^{-3}$ in flat frequency channel. Here, $N=\tilde{N}=16$, $M=\{2,4\}, \tilde{M}=\{4,16\}$. The bias used for numerical simulations are indicated with each SE/EE coordinate.

suitable for low alphabet cardinalities. Thus, we identify a region of interest (ROI) (cf. Fig. 4) for the proposed approach. Intuitively, lower EE of DCO-FOFDM-IM for higher alphabet cardinalities can be explained by the fact that the SAP is determined using $N$ subcarriers (rather than $N / 2-1$ subcarriers as in DCO-OFDM-IM), resulting in higher number of errors in index identification which propagate to the modulation bits errors resulting in higher BER.

Fig. 5 compares BER performances in both flat frequency and time dispersive channels for same SE, i.e., $2 \mathrm{bits} / \mathrm{s} / \mathrm{Hz}$. SE of $2 \mathrm{bits} / \mathrm{s} / \mathrm{Hz}$ is obtained when $\{\tilde{M}, \tilde{N}, \tilde{K}, \beta, \tilde{\psi}\}=\{4,32,11,6,0.6875\}$ for DCO-OFDM$\mathrm{IM},\{M, N, K, \beta, \psi\}=\{2,32,9,5,0.2812\}$ for DCOFOFDM-IM, and $\{M, N, \beta\}=\{2,16,5\}$ for DCO-FOFDM. The impulse response for time dispersive channel is $h(t)=$ $\zeta \frac{6 \kappa^{2}}{(t+\kappa)^{7}} u(t)$, where $\kappa=2 H / c, H$ is the ceiling height, $c$ is the speed of light, $\zeta$ is the optical path loss normalized to $1, \Delta \tau=(\kappa / 12) \sqrt{13 / 11}$ is the root-mean-square (rms) delay spread of the channel which is set to $10 \mathrm{~ns}$, and $u(t)$ is the unit step function. For time dispersive channel, the data rate is $50 \mathrm{Mbits} / \mathrm{s}$, a cyclic prefix of length $0.25 \mathrm{~N}$ (resp. $0.25 \tilde{N})$ and zero-forcing equalization is used. In Fig. 5, low $E_{\mathrm{b}_{(\text {elec) }}} / N_{0}$ region depicts the BER performances in flat fading channel, whilst the high $E_{\mathrm{b}_{\text {(elec) }}} / N_{0}$ region illustrates the BER performances in time dispersive channel. Observe that the BER performance of DCO-FOFDM-IM is better than others in both flat frequency and time dispersive channels because: (i) lower sparisty factor alleviates the filtering impact of the time dispersive channel and leads to a lower bias requirement since the PAPR is reduced; and (ii) a higher signal to clipping noise power ratio per subcarrier for IM approaches relative to non-IM approaches.

Considering maximum achievable SE in the ROI, the practicality of DCO-FOFDM-IM is apparent from the fact that to attain data rate of $50 \mathrm{Mbits} / \mathrm{s}$, the base-band bandwidth needed is $17.39 \mathrm{MHz}$ when $\{M, N\}=\{2,16\}$ in comparison to $28.57 \mathrm{MHz}$ for DCO-OFDM-IM with $\{\tilde{M}, \tilde{N}\}=\{4,16\}$, and $25 \mathrm{MHz}$ for DCO-FOFDM with $\{M, N\}=\{2,16\}$. The bandwidth demands for DCO-OFDM-IM and DCO-FOFDM

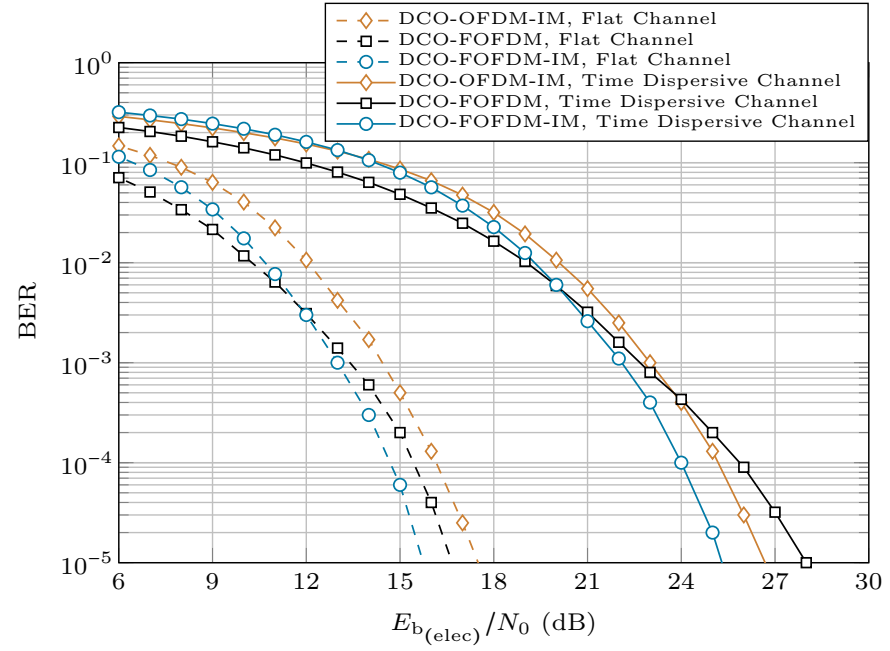

Fig. 5: BER performance comparison of DCO-FOFDM-IM with other benchmarks for SE of $2 \mathrm{bits} / \mathrm{s} / \mathrm{Hz}$ in both flat fading and time dispersive channels. may exceed the bandwidth of light-emitting diodes which is a few tens of MHz. Furthermore, the chip duration for DCOFOFDM-IM is $\approx 0.0288 \mu \mathrm{s}$, whereas for DCO-OFDM-IM and DCO-FOFDM, it is $\approx 0.0175 \mu \mathrm{s}$ and $\approx 0.0200 \mu \mathrm{s}$, respectively. In a nutshell, the above-mentioned analysis dictates that DCO-FOFDM-IM needs lesser bandwidth and low speed converters compared to other schemes.

\section{CONCLUSION}

In this letter, we investigate DCO-FOFDM-IM for OWS and demonstrate its advantages over classical benchmarks. Its foremost advantage is its ability to realize higher achievable $\mathrm{SE}$; and extended granularity with a better EE/SE trade-off in ROI for low modulation orders, in flat frequency channel. For time dispersive channels, still considering low modulation orders, DCO-FOFDM-IM exhibits improved resistance to dispersion due to the inherent sparsity of the signal. Furthermore, DCO-FOFDM-IM allows relaxed requirements for the converters and bandwidth. The advantages of DCO-FOFDMIM over classical approaches identified hereby make it a viable candidate for OWS.

\section{REFERENCES}

[1] J. Zhou, Y. Qiao, T. Zhang, E. Sun, M. Guo, Z. Zhang, X. Tang, and F. Xu. FOFDM based on discrete cosine transform for intensity-modulated and direct-detected systems. J. Lightw. Tech., 34(16):3717-3725, 2016.

[2] P. A. Haigh and I. Darwazeh. Visible light communications: Fastorthogonal frequency division multiplexing in highly bandlimited conditions. In Intl. Conf. Commun. in China, pages 1-8, 2017.

[3] E. Basar, M. Wen, R. Mesleh, M. Di Renzo, Y. Xiao, and J. Haas. Index modulation techniques for next-generation wireless networks. IEEE Access, 5:16693-16746, 2017.

[4] T. Mao, Q. Wang, Z. Wang, and S. Chen. Novel index modulation techniques: A survey. IEEE Commun. Surveys Tuts., 21(1):315-348, 2018.

[5] E. Başar and E. Panayırci. Optical OFDM with index modulation for visible light communications. In Intl. Wksp. Opt. Wireless Commun., pages 11-15, 2015.

[6] S. Mazahir, A. Chaaban, H. Elgala, and M.-S. Alouini. Achievable rates of multi-carrier modulation schemes for bandlimited IM/DD systems. IEEE Trans. Wireless Commun., 18(3):1957-1973, 2019.

[7] M. Chafii, J. P. Coon, and D. A. Hedges. DCT-OFDM with index modulation. IEEE Commun. Lett., 21(7):1489-1492, 2017.

[8] P. K. Frenger and N. A. B. Svensson. Parallel combinatory OFDM signaling. IEEE Trans. Commun., 47(4):558-567, 1999.

[9] J. B. Carruthers and J. M. Kahn. Modeling of nondirected wireless infrared channels. IEEE Trans. Commun., 45(10):1260-1268, 1997. 